Europhysics Letters

PREPRINT

\title{
Log-Poisson statistics and full aging in glassy systems
}

\author{
PaOlo Sibani and Jesper Dall \\ Fysisk Institut, Syddansk Universitet-5230 Odense M, Denmark
}

PACS. 05.40.-a - Fluctuation phenomena, random processes, noise, and Brownian motion.

PACS. 75.10.Nr -Spin-glass and other random models.

PACS. 65.60.+a - Thermal properties of amorphous solids and glasses.

\begin{abstract}
We argue that Poisson statistics in logarithmic time provides an idealized description of non-equilibrium configurational rearrangements in aging glassy systems. The description puts stringent requirements on the geometry of the metastable attractors visited at age $t_{w}$. Analytical implications for the residence time distributions as function of $t_{w}$ and the correlation functions are derived. These are verified by extensive numerical studies of short range Ising spin glasses.
\end{abstract}

Introduction. - Aging is a key aspect of the dynamics in a host of complex glassy systems [1, 2, 3, 4, 5, 6, 7, 8, Its main characteristics are a slow, sub-linear drift of macroscopic averages with the age $t_{w}$ of the system, i.e. the time elapsed after an initial thermal quench, and a concomitant age dependence of the correlation and response functions. The latter shows that a quasi-stationary fluctuation regime for short observation times $t<t_{w}$ is followed by off-equilibrium dynamics for $t>t_{w}$. This behavior can be attributed to an increasing degree of thermal stability of the dynamically relevant attractors or landscape valleys as the age $t_{w}$ grows $9,10,11,12$.

Within each valley, the pseudo-equilibrium fluctuations are determined by the free energy. However, the inter-valley non-equilibrium dynamics could be practically irreversible, and hence qualitatively different. Adopting the simplified view that the selection is truly irreversible allows us to formulate a theoretical description of large non-equilibrium rearrangements, or 'quakes', which borrows from studies of non-thermal models [13 14] and which supplies a basis to the striking similarity of complex dynamics seen in very different systems with multiple metastable states. The key feature is dynamical invariance to shifts in the logarithm of time. This implies that the time spent in thermally metastable attractors is power-law distributed with an exponent $\alpha$. Furthermore, the distribution has a $t / t_{w}$ aging behavior, often called full or pure aging. The exponent $\alpha$ depends on the system size but not on the temperature. The further assumption that the memory of the initial configuration decays as $\exp (-\gamma k)$ with the number $k$ of quakes leads to a power-law decay and a $t / t_{w}$ form of the correlation function for $t>t_{w}$. The corresponding non-equilibrium exponent $\lambda$ is jointly determined by $\alpha$ and $\gamma$.

These theoretical results are checked through extensive numerical simulations of short ranged spin glass systems. While direct experimental evidence is not yet available, possibly related large-scale irreversible rearrangements similar to quakes have recently been identified in aging colloidal gels [8].

(c) EDP Sciences 
Basic formalism. - Beyond its original macro-evolutionary context, the concept of 'punctuated equilibrium' [15] is applicable to any stochastic dynamics where long periods of stagnation are dotted by fast irreversible changes. This occurs e.g. in micro-evolution [16 17. and in certain driven dissipative models 13, 14. Within a population evolving in a fitness landscape [17, fitness records achieved by an individual through random mutations are amplified to the macroscopic level by darwinistic selection and trigger punctuations. Since the same statistics describes both punctuations and records, the analytical description available for the latter 13 can be applied to the former. Below, we discuss how thermal noise may similarly induce large and irreversible quakes in glassy systems.

To summarize the basic ideas of record statistics [13, consider times $t_{w} \geq 1$ and $t \geq 0$, and independently draw for each integer time a random number from any fixed distribution not supported on a finite set. The attempts whose outcome exceeds all previous outcomes are marked as records. For large times, the probability that $k$ such events occur in $\left[t_{w}, t_{w}+t\right]$ is then

$$
P_{k}\left(t_{w}, t_{w}+t\right)=\frac{1}{k !}\left[\alpha \log \left(\frac{t_{w}+t}{t_{w}}\right)\right]^{k}\left[\frac{t_{w}+t}{t_{w}}\right]^{-\alpha},
$$

where $\alpha=1$ for a single sequence of attempts. The total number of records produced by $n$ independent sequences is a sum of log-Poisson variables, and hence itself log-Poisson distributed, with $\alpha=n$.

Equation (11) can be recognized as a Poisson distribution with $\log \left(t_{w}+t\right)-\log \left(t_{w}\right)$ replacing the linear difference $t$ between the time arguments, or, for short, a log-Poisson distribution $\left({ }^{1}\right)$. Equation (11) implies that the rate of events decays as $\alpha /\left(t+t_{w}\right)$, while the logarithmic rate of events approaches the constant value $\alpha$ for $t \gg t_{w}$.

Glossing over the discreteness of the time arguments, we can now pick thermal noise as the (arbitrary) source of randomness producing the records. Since landscape induced correlations are negligible if records are intercalated by long intervals spent dwelling near a local energy minimum, the number of energy records observed in a fixed time interval is expected to be proportional to a log-Poisson variable [18. The same applies if energies are measured relative to the current lowest energy minimum, producing the energy difference henceforth referred to as a 'barrier'. With this definition, not all barrier crossings carry physical significance. However, if quakes are log-Poisson distributed, they are naturally associated with the crossing of barriers of record magnitude. Two special landscape features sketched in Fig 1 would allow barrier records to induce quakes: i) The stability of the attractors successively selected, as gauged by e.g. an exit barrier, must only increase marginally [13.14], i.e. in principle by an infinitesimal amount. This requires that shallow attractors be predominant, and implies that the actual magnitude of the records generating the quakes is immaterial $\left({ }^{2}\right)$. ii) A large and negative energy change associated to a quake, ensures its irreversibility. Such changes are observed in both atomistic glass models [20] and spin glasses [18],

The only adjustable quantities in this landscape cartoon are the parameter $\alpha$ and the form of the quake induced decorrelation. The noise independence of the statistics restricts $\alpha$ to be a temperature independent quantity. An interesting increase with system size can arise in spatially extended systems, where sufficiently distant regions can evolve independently.

In the sequel, we first deal with some theoretical consequences of the marginal stability of the attractors and irreversibility of the quakes. To make contact with experiments, we then

$\left({ }^{1}\right)$ The equation was derived in 13 for $t_{w}=1$, but the generalization to the propagator $P_{n}\left(t_{w}, t_{w}+t\right)$ is straightforward and completely analogous to the standard case.

$\left({ }^{2}\right)$ The predominance of shallow attractors concurs with the observation that thermal quenches produce shallow minima in glassy systems, and fits the anomalous sensitivity of complex relaxation to small mechanical or thermal perturbations implied by the so-called reset or memory effects [19]. 


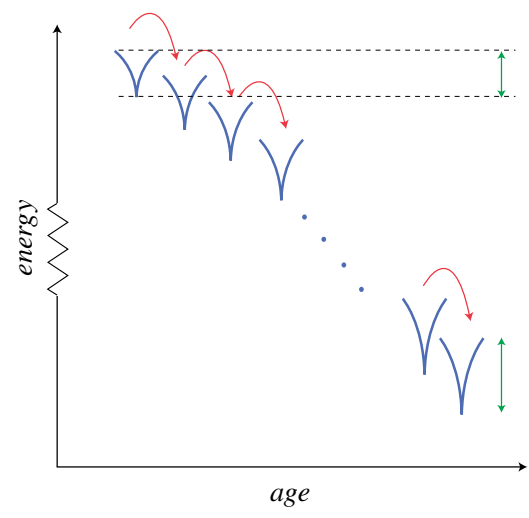

Fig. 1

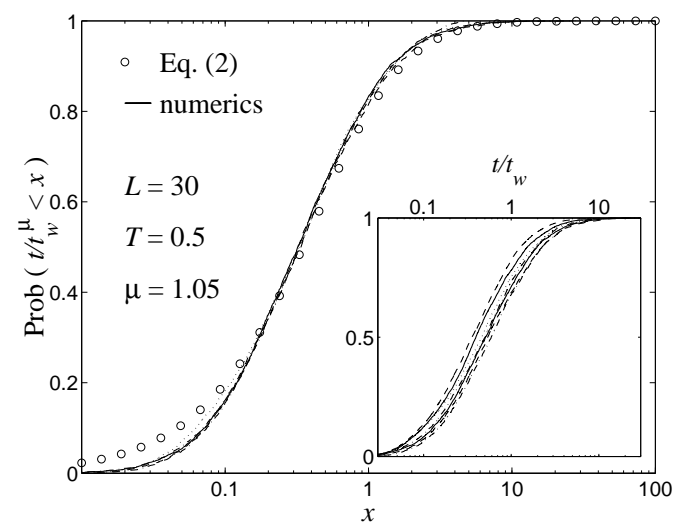

Fig. 2

Fig. 1 - An idealized landscape where the attractors are symbolized by wedges. The arrows represent 'quakes' leading from one attractor to the next. They entail a large energy change - the vertical displacement - and are therefore irreversible. The log-Poisson statistics of the quakes originates from the infinitesimal increase of the metastability of the attractors selected, which is rendered by a slight increase in the bottom to top distance (depth) of each trap.

Fig. 2 - The shown curves all describe the distribution of a suitably rescaled residence time in the attractors - or valleys - of the sort sketched in Fig. 11 The circles are the prediction of the log-Poisson theory-Eqs. (1) and (2) - and correspond to full aging $(\mu=1)$. The empirical data for the spin glass landscape are plotted as lines, and describe the scaled residence time $t_{r} / t_{w}^{\mu}$ in valleys entered at $10^{2} \leq t_{w} \leq 10^{5}$. They merge into a seemingly continuous line due to the quality of the data collapse. The insert shows the same data with the less satisfactory $\mu=1$ scaling.

summarize an empirical way to identify quakes in glassy systems [18 and apply it to spin glasses.

Attractor residence times. - The distribution of the residence or trapping time $t_{r}$ in valleys of an energy landscape has been considered theoretically 11 12, but has not previously been measured.

Let $t_{k}$ mark the occurrence of the $k$ 'th event in a log-Poisson process with logarithmic rate $\alpha$. In perfect analogy to the usual Poisson process, the ' $\log$-waiting time' $\log \left(t_{k}\right)-\log \left(t_{k-1}\right)$ has the exponential distribution

$$
\operatorname{Prob}\left(\log \left(t_{k} / t_{k-1}\right)<x\right)=1-\exp (-\alpha x)
$$

which is mathematically equivalent to the event distribution given in Eq. (1). We now consider the distribution of the residence time $t_{r}=t_{k}-t_{k-1}$ for traps entered at $t_{k-1}=t_{w}$. As $t_{r} / t_{w}=t_{k} / t_{k-1}-1$, we find

$$
\operatorname{Prob}\left(t_{r} / t_{w}<x\right)=1-(x+1)^{-\alpha}
$$

which corresponds to full aging behavior. Equation. (2) with $\alpha=2.3$ is plotted (circles) in the main panel of Fig. 2 together with spin glass data (lines). These are scaled in a slightly different manner, to account for a small but systematic deviation from full aging.

The power-law form of Eq. (2) was suggested by Bouchaud 12, with the added and crucial assumption of an infinite average residence time. In our case, the average residence time $\left\langle t_{r}\right\rangle$ 
is equal to $t_{w} /(\alpha-1)$ which is finite and $\approx t_{w}$ for the observed $\alpha \approx 2$. As a consistency check, we note that the largest relaxation time $\tau_{e q}$ in a valley must be smaller than $\left\langle t_{r}\right\rangle$, for local equilibration to apply. When the latter is of order $t_{w}, \tau_{e q}<t_{w}$ follows. In the lack of special symmetries [21], $\tau_{e q}=\tau_{c o r r}$, where $\tau_{c o r r}$ characterizes the short time decay of correlation functions, either as a cut-off parameter or directly as the time constant of an exponential decay. Hence $\tau_{c o r r}<t_{w}$ as expected.

Scaling of correlations. - Additional information on the effect of the quakes enters the calculation of thermally averaged correlations functions. Let $c(m ; m+k)$ be the configurational overlap between the lowest energy configurations in valley $m$ and $m+k$. With probability $P_{m}\left(1, t_{w}\right) P_{k}\left(t_{w}, t_{w}+t\right), m$ and $m+k$ are the 'current' attractors at times $t_{w}$ and $t_{w}+t$, whence the non-equilibrium part of the configurational autocorrelation function is given by

$$
\bar{C}\left(t_{w}, t_{w}+t\right)=\sum_{m=1}^{\infty} \sum_{k=0}^{\infty} c(m ; m+k) P_{m}\left(1, t_{w}\right) P_{k}\left(t_{w}, t_{w}+t\right) .
$$

Of special interest is $c(1 ; 1+k)$, the average overlap between the first and the $(1+k)^{\prime}$ th valley. This is the sole contribution to Eq. (3) for $t_{w}=1$, in which case $\bar{C}(1,1+t)$ additionally describes the magnetization decay of the fully polarized configuration of a spin glass [22. The exponential form

$$
c(1 ; 1+k)=\left(1-c_{\infty}\right) e^{-\gamma(T) k}+c_{\infty}
$$

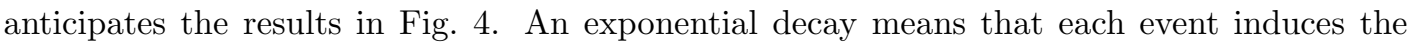
same relative change, and leads to

$$
\bar{C}(1,1+t)=\sum_{k=0}^{\infty} c(1 ; 1+k) P_{k}(1,1+t)=\left(1-c_{\infty}\right) t^{-\lambda}+c_{\infty}
$$

where the non-equilibrium exponent $\lambda(T)$ is given by

$$
\lambda(T)=\alpha\left(1-e^{-\gamma(T)}\right) \approx \alpha \gamma(T)
$$

Equation (6) thus links a dynamical exponent to the landscape geometry. The second approximate equality given in Eq. (6) is only justified for $\gamma \ll 1$.

The further assumption that $c(m ; m+k)$ is altogether independent of $m$ amounts to translational invariance with respect to $m$, and hence with respect to additive shifts in the logarithm of $t_{w}$. These are equivalent to a multiplicative rescaling of $t_{w}$. Not surprisingly, Eq. (3) yields the scale invariant and full aging form

$$
\bar{C}\left(t_{w}, t_{w}+t\right) \propto\left(\frac{t+t_{w}}{t_{w}}\right)^{-\lambda} .
$$

Landscape exploration. - How should valleys and quakes be concretely defined given a time sequence of data? One approach is the protocol developed [18 for numerical data describing isothermal relaxation after a deep quench. The basic observation is that at sufficiently low $T$ pseudo-equilibrium fluctuations repeatedly visit the same local energy minimum. Hence, the attainment of an energy value lower than all previous values implies a non-equilibrium event, i.e. an ongoing quake. Several closely spaced minima records will often occur as a quake slides downhill among which the one preceding a barrier record is likely to be the most physically significant, as it - ideally - coincides with a new local minimum. By the same token, in 
a series of barrier records corresponding to an uphill climb, the last record preceding a low energy record is most significant, as it marks the access to a new local minimum, and hence the start of a quake. The times at which the above types of event occur provide an empirical definition of both valleys and quakes.

This procedure yields a null result if i) only a single energy minimum is present; or ii) the initial condition is the ground state irrespective of the number of local minima present; or iii) all the minima and/or barriers are equal. Also, iv) if the trajectories can bypass the energy barriers and edge their way into states of lower energy through 'entropic barriers', the number of valleys discovered remains less or equal to one at all times.

The actual outcome is very different, as illustrated in Fig. 3. where the estimated average and variance of the number $n_{V}$ of valleys visited is plotted versus the logarithm of time. An equality between variance and average is a key feature of Poisson distributions. If we neglect the initial curvature, the estimated quantities are very nearly proportional to $\log t$, indicating that $n_{V}$ itself is proportional to a log-Poisson variable through a constant of order one. We emphasize that log-Poisson statistics is an idealized low $T$ description, and that systematic corrections in the form of a decaying logarithmic rate of events must be expected, since each valley has a growing probability to contain the true ground state. Since $\alpha$ is not truly constant, the single value required by the log-Poisson theory cannot be estimated accurately.

As $T$ grows, local thermal equilibration within each attractor is not granted, and low energy records lose connection to landscape minima. A check of the energy and configurational changes associated with the quakes, of the sort performed in [18 is required to independently establish their physical relevance. We additionally checked that the low energy record configurations are close (albeit usually not identical) to actual valley minima.

Numerical results. - We performed extensive simulations of short-ranged Ising spin glasses on cubic lattices ( $L^{3}$ spins) with periodic boundary conditions and symmetric Gaussian couplings with unit variance, see also [18. The Waiting Time Method [23] with single spin flip dynamics, which is dynamically equivalent to the Metropolis algorithm but much faster at low $T$, was used. The 'intrinsic' and size independent time variable of this method corresponds to the number of Monte Carlo (lattice) sweeps in the Metropolis as well as to the physical time of a real experiment. In all our runs, we skip the data within the first 10 time units in order to let the system settle down from its random $T=\infty$ initial configuration.

The set of data shown in Fig. [3 was obtained for a system of linear size $L=30$ at temperature $T=0.5$. Numerous similar plots (not shown) were obtained for temperatures in the range $0.2 \leq T \leq 0.8$, for $8 \leq L \leq 30$ and $t_{w} \leq 10^{6}$. In most cases 20000 independent runs were performed for each value of $T$ and $L$, removing any visible statistical flutter. When $\alpha$ is calculated as the logarithmic slope of the number of valleys seen on average in the time window $\left[10^{4}, 10^{6}\right]$, its size dependence for $L=13,16,20$ and 30 is found to be $\alpha=1.17(1)$, $1.37(1), 1.58(1)$ and $1.63(1)$, respectively. While the growth clearly tapers off, we cannot with confidence conclude on the true asymptotic behavior for large $L$. We note, however, that $\lambda(T) \approx \alpha \gamma(T)$ should remain finite in the thermodynamic limit. Hence, the growth of $\alpha$ must either remain bounded, or be compensated by a corresponding decrease of $\gamma(T)$. To see that the latter is likely the case, we note that $\gamma=-\log \left(1-2 H_{d} / L^{d}\right)$, where $H_{d}$ is the Hamming distance between the local minima at the bottom of consecutive $(k=1)$ valleys. In $3 \mathrm{~d}, H_{d} \propto L^{2.8}$ was found in [18, whence, using $H_{d} / L^{d}<<1$ one obtains the slow decay $\gamma \propto L^{-0.2}$.

In order to obtain a proper log-Poisson variable, the number of valleys must be divided by $\approx 0.7$, the ratio between variance and average, and the empirical value of $\alpha$ must be similarly rescaled to the effective value $\alpha \approx 2.0$ for a $16^{3}$ system, and $\alpha \approx 2.3$ for a $30^{3}$ system. 
EUROPHYSICS LETTERS

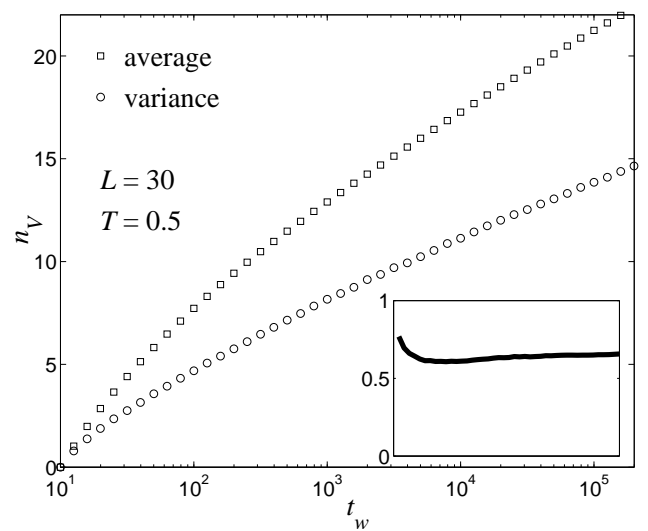

Fig. 3

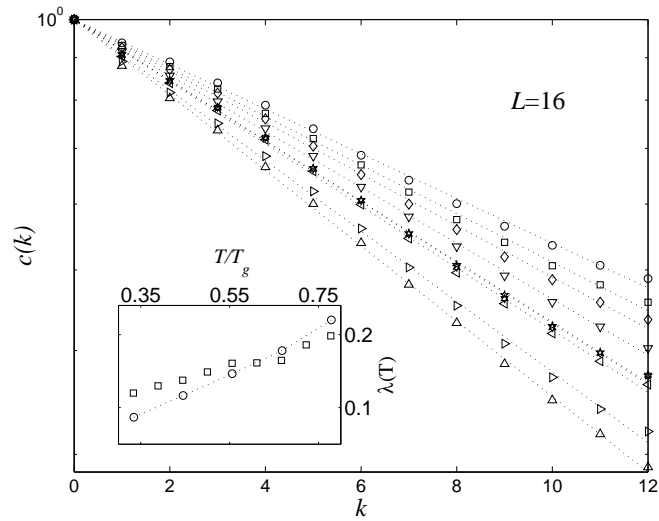

Fig. 4

Fig. 3 - The average and variance of the number of valleys $n_{V}$ visited in time $\left[10, t_{w}\right]$. Their ratio is shown as a function of time in the insert. After a short transient, average and variance are nearly proportional to one another and to $\log t_{w}$, indicating that $n_{V}$ is proportional to a log-Poisson variable. In the time interval $\left[10^{4}, 10^{6}\right]$, the logarithmic slope is $\alpha=1.63(1)$.

Fig. 4 - The main panel shows that the assumed exponential form of $(c(k)-c(\infty)) /(1-c(\infty))$ versus $k$ is supported by the data, for temperatures $T=0.30$ (upper curve) $0.35,0.40 \ldots 0.70$. The lines are obtained by a two-parameter fits, and the symbols correspond to data points. The insert shows the temperature dependence of the non-equilibrium exponent $\lambda\left(T, t_{w}=10\right)$ according to [22] (circles) and as given by Eq. (6) with $\alpha=2.0$. The age argument $t_{w}=10$ was chosen for the comparison, as it corresponds to the earliest time at which our data were collected. All temperatures are divided by $T_{g}=0.9$.

Next, we analyze the distribution of the residence time $t_{r}$ within valleys entered at time $\left[0.95 t_{w}, 1.05 t_{w}\right]$ for $10^{2} \leq t_{w} \leq 10^{5}$. The observed frequencies of the scaled quantities $t_{r} / t_{w}^{\mu}$ with $\mu=1.05$ are obtained from the same runs as in Fig. 3] They are plotted as lines in the main panel of Fig. 2 and the excellent quality of the resulting collapse gives the false impression of a single continuous line. The same distributions are also plotted in the insert, this time with $\mu=1$, which is the theoretical prediction of the log-Poisson statistics. We see that while $\mu=1$ fails to produce a complete data collapse, it nevertheless accounts for the main features of the distribution. The corresponding theoretical prediction of Eq. (2) with $\alpha=2.3$ is given by the circles in the main panel.

We finally consider the correlation decay. The overlap $c(1 ; k+1)$-for short $c(k)$ - between the lowest lying configurations of the first and the $(1+k)$ 'th valley was calculated separately for temperatures $T=0.3,0.35, \ldots, 0.7$ starting in each case from a random initial condition. In this case, data are for each $T$ averages over 2000 trajectories belonging to 20 different realizations of the couplings. Since no data were taken for the first 10 time steps, the smallest age value considered is $t_{w}=10$, rather than the theoretical value $t_{w}=1$. A two parameter non-linear fit to the exponential form in Eq. (4) was performed, producing the lines shown in the main panel of Fig. 4 while the actual data are given by the symbols. The main error in fitting $c(k)$ is systematic and originates from the poor sampling of late events, which is dealt with by introducing a cut-off at $k=12$. The fitted values of $\gamma$ and $c_{\infty}$ vary a few percent as the position of the cut-off is varied slightly around this value. The insert of Fig. 4 compares the temperature dependence of the predicted non-equilibrium exponent $\lambda \approx \alpha \gamma(T)$ (squares) 
with the corresponding result by Kisker et al. 22. The comparison is primarily qualitative, since we are unable to determine $\alpha$ with great precision. We used $\alpha=2.0$ in this plot.

The (implied) temperature dependence of $\gamma(T)$ shows that, for the same number of quakes, configurations decorrelate faster at higher temperatures, as one would expect. We note in passing that $c_{\infty}$ systematically decreases with $T$, and that $c_{\infty}$ would equal the EdwardsAnderson order parameter if the simple exponential decay of $c(k)$ were to continue indefinitely.

While the exponential decorrelation form given by Eq. (7) - and the self-similar picture of the landscape it implies - is required for full aging, it is is not likely to be exactly fulfilled on all time scales. Yet it agrees with the full aging behavior observed in spin glasses 24, which highlights the dynamical nature of their behavior. Interestingly, strong superaging behavior is found in gels [8]. Hence, either the latter systems are closer to thermal equilibrium than spin glasses, or considerable deviations from the marginal stability increase of the visited attractors occur.

This project has been supported by Statens Naturvidenskabelige Forskningsråd through a block grant and by the Danish Center for Super Computing with computer time on the Horseshoe Linux Cluster. We are grateful to S. Boettcher and to J.C. Schön for useful comments and discussions.

\section{REFERENCES}

[1] Reim W., Koch R. H., Malozemoff A. P., Ketchen M. B., and Maletta H., Phys. Rev. Lett, 57 (1986) 905.

[2] Refrigier P and Ocio M., Revue Phys. Appl., 22 (1987) 367.

[3] Andersson J-O., Mattsson J. and Svedlindh P., Phys. Rev. B, 46 (1992) 8297.

[4] Nicodemi M. and Jensen H. J., J. Phys A, 34 (2001) 8425.

[5] Bureau L., Baumberger T. and C. CAroli., cond-mat/0202245, 2002.

[6] Hannemann A., Schön J. C., M. Jansen M. and Sibani P., cond-mat/0212245 2002.

[7] Kityk A. V., Rheinstädter M. C., Knorr K., and Rieger H., Phys. Rev. B, 65 (2002) 14415.

[8] Bissig H., Romer S., Cipelletti L., Trappe V., and Schurtenberger P., PhysChemComm, 6 (2003) 21.

[9] Sibani P. and Hoffmann K. H.,. Phys. Rev. Lett., 63 (1989) 2853.

[10] Hoffmann K. H. and Sibani P., Z. Phys. B, 80 (1990) 429.

[11] Vincent E., Bouchaud J.-Ph, Dean D. S. and Hammann J., Phys. Rev. B, 52 (1995) 1050.

[12] Bouchaud J-Ph., J. Phys. I France, 2 (1992) 1705.

[13] Sibani P. and Littlewood P. B., Phys. Rev. Lett., 71 (1993) 1482.

[14] Sibani P. and Andersen C. M., Phys. Rev. E, 64 (2001) 021103.

[15] Gould. S and Eldredge N.,. Paleobiology, 3 (1977) 115.

[16] Lenski R. and Travisano M., Proc. Natl. Acad. Sci., 91 (1994) 6808.

[17] Sibani P. and Pedersen A., Europhys. Lett., 48 (1999) 346.

[18] Dall J. and Sibani P., cond-mat/0302575 2003.

[19] Berthier L and Bouchaud J.-Ph., Phys. Rev. B, 66 (2002) 054404.

[20] Schön J. C. and Sibani P., Europhys. Lett., 49 (2000) 196.

[21] Van Kampen N. G., Stochastic Processes in Physics and Chemistry. (North Holland) 1992.

[22] Kisker J., Santen L., Schreckenberg M. and Rieger H., Phys. Rev. B, 53 (1996) 6418.

[23] Dall J. and Sibani P., Comp. Phys. Comm., 141 (2001) 260.

[24] Rodriguez G. F., Kenning G. G. and Orbach R., cond-mat/0212259 2002. 